\title{
What counts as success in genetic counselling?
}

\author{
Ruth F Chadwick University of Wales, Cardiff
}

\section{Author's abstract}

The question of what counts as a successful outcome of the process of genetics counselling has recently become central because of the increasing calls for efficiency in health care, and for means of measuring efficiency. Angus Clarke has drawn attention to this trend, and has argued against both a measure in terms of the number of terminations of pregnancy performed as a result of counselling, and an assessment in terms of the contribution of genetics counselling to a national eugenics policy. He suggests instead a measure of workload.

There are good arguments for supporting Clarke's position up to a point. In looking for an appropriate measure, much turns on how genetics counselling is defined. It is here understood in the context of an autonomy model of health care. It is argued that there is a contradiction between such an interpretation and the termination measure of outcome. The political philosophy underlying this outcome is also defective.

Workload is not sufficient as a measure of outcome however; it is essential to look at the proper goals of an activity. It is argued that these must be connected in some way with the genetic health of the population; that the promotion of reproductive autonomy itself is not sufficient as a goal. The concern for genetic health, however, is interpreted in a way that avoids Clarke's concerns about a national eugenics policy.

The question of what counts as a successful outcome of genetics counselling has recently become critical because of increasing calls for efficiency in health care. Angus Clarke in the Lancet (1) has drawn attention to this trend and to the possibility of a desire, coming from management, to measure the efficiency of a medical genetics unit in terms of the number of terminations performed as a result of genetics counselling. His additional concern is that a medical genetics unit might be assessed in terms of its contribution to a national eugenics policy. He suggests instead that efficiency could be measured in terms of workload.

\section{Key words}

Genetic counselling; autonomy; eugenics.

\section{What is genetic counselling?}

Much turns on what genetics counselling is. Clarke is anxious to distinguish counselling from advice. $\mathrm{He}$ writes that whereas advice is a 'prescriptive activity, often subtly authoritarian', genetics counselling is 'informative, supportive and "enabling"' (2). It supports people in reaching their own decisions.

'Genetics counselling' I take to include the following kinds of activity: (a) advising adults preconception, of the probability of their conceiving a child suffering from a genetic disorder; (b) advising adults, post-conception, and as a result of some method of fetal screening, as to whether or not a fetus is suffering from a genetic disorder; (c) alerting them to the options open to them.

This account, I think, avoids any suggestion that genetics counselling involves telling people what option they ought to choose. It is not, however, intended to be an exhaustive account of what genetics counsellors do. It omits, for example, the notion of counselling as helping people to live with the consequences of genetic disorders and of their decisions.

So how can we judge its success?

\section{Medical audit and number of terminations}

The pressures towards a termination measure are obvious: such an approach would give an outcome that could be measured in numerical terms. Further, the number of terminations could be translated into a figure representing the expected financial saving to the health service of the amount of money that would have been needed to provide care for a child with a genetic disorder.

It may be that we feel an intuitive repugnance towards accepting such a measure of success for a medical genetics service, but we need to look at whether there are any good arguments for opposing it. It will be argued by some that the aim of any medical service ought not to be the termination of life, but this argument will not do here if it is accepted that offering terminations is one acceptable response to the finding of genetic disease. I shall not argue this here. For the purposes of this article I shall 
assume that there are at least some cases in which termination is a morally acceptable outcome.

Clarke's objection to the use of this particular outcome measure is that it would put 'subtle - and possibly less than subtle - pressure upon clinicians to maximise the rate of terminations for "costly" disorders' (2) by, for example, persuading possibly reluctant couples to choose termination.

There is clearly a risk of undesirable consequences for the client-counsellor relationship of using the particular outcome measure outlined. However, there is a further, logical point. There is a contradiction between the proposed outcome measure and the above account of genetic counselling, in terms of facilitating choice.

This was in accordance with respecting the autonomy of clients, respecting their right to choose. If the measure of success is in terms of one particular decision about outcome, how can this be compatible with the autonomy model of health care?

The contradiction only exists if the belief in the value of the autonomy model and the belief in the value of one particular outcome measure are held simultaneously. Perhaps those who put forward the outcome measure do not themselves subscribe to the autonomy model and are suggesting that it itself has to give way.

Interestingly, though, the outcome measure proposal comes from the same stable, politically speaking, as belief in autonomy as a political value. The drive towards what is described as a more efficient health service goes hand in hand with the purported upholding of consumer choice, underpinned by an expressed belief in the value of autonomy.

I have argued in another context (3) that this political ideology in fact distorts the concept of autonomy; that a value whose rationale is to support the freedom of choice of the individual against the power of government and professionals has been brought into the service of the promotion of selfreliance. Being autonomous, rather than meaning self-determining, comes to mean standing on your own two feet, so that a rationalisation is provided for cutting services while apparently upholding freedom of choice, even though in fact the cuts diminish choice.

How does this apply in the present context? I am suggesting that in order to see if there is any contradiction between the advocating of the outcome measure and the upholding of the autonomy model, it is necessary to look at the meaning of autonomy involved. While there may be a contradiction between a belief in freedom of choice and measuring outcomes in terms of one particular decision, the same does not apply if what is valued under the name of autonomy is self-reliance. Both advocate less dependence on public funding for those suffering from genetic disorders.

This makes it clear that if the outcome measure is found unacceptable, it is not just this that has to be argued against, but also the political philosophy that underpins both it and a certain interpretation of autonomy. I would argue further that it should be argued against precisely because it does distort the meaning of autonomy for the purpose of denying public responsibility for those in need.

\section{Eugenics}

If not the number of terminations, then what? Clarke suggests a workload audit (4). While a satisfactory workload may be a necessary condition of a service's being successful, however, it is not sufficient. It is necessary to know not just how much work is being done but whether that work is meeting objectives, and those objectives need to be specified. Clarke is anxious to avoid the suggestion that what a medical genetics unit is about is eugenics. He asks, "Are we concerned with the "genetic health" of the population, of the race? Or are we instead concerned with the concrete individual or family sitting in front of us now, and with providing information and support for them' (5)?

Clarke recognises, rightly, that there is no necessary contradiction here. To decide that one's objectives are concerned with the genetic health of the population is not incompatible with a decision to achieve that by means which show respect and concern for individual clients. But he is worried that conflicts of interest may arise.

The supposed conflict of interest again arises from the worry that if government takes an interest in the genetic health of the population, there may be pressure on individuals to make certain sorts of reproductive decisions.

There are, also, quite widespread fears about potential undesirable longer term consequences of advances in genetic-screening programmes, such as job discrimination against people who are thought to have undesirable genes. Now discrimination on the basis of genes alone is as unjustifiable as discrimination on the basis of race or sex, and must be argued against on the same grounds. Nothing follows from this, however, about the desirability or otherwise of a medical genetics unit being concerned with the incidence of genetic disease in the population as a whole. There are reasons for wanting to reduce the incidence of genetic disease and these are connected with the consequences of genetic diseases for their sufferers.

In fact if medical geneticists do not hold views of this nature, it is difficult to see how they can justify their service, unless they do fall into the trap of arguing in terms of money-saving, in the way outlined above.

In an influential article, Bernard Williams (6) drew a distinction between internal and external goals of an activity. An internal goal is one to which the activity is logically connected; an external goal is 
one to which the activity is only contingently linked. As a matter of logic the goal of a medical genetics service must be connected in some way with the incidence of genetic disease, whether this is expressed in a negative way, in terms of a reduction in the incidence of genetic disease, or in a positive way, in terms of promotion of genetic health. The very fact that geneticists think it desirable to offer their service to individuals shows that there is at least a presumption that it is undesirable to suffer from genetic disease and that means should be offered of avoiding it.

The concern that Clarke has about its being seen as a eugenics service arises from a confusion about ends and means. The worry arises from the possibility that clinicians will be given targets to fulfil in accordance with a national eugenics policy. He says:

‘. o our funding might depend upon "units of handicap prevented", which might pressurise parents into screening programmes and then into unwanted terminations with the active collusion of clinical geneticists anxious about their budgets. Such targets could well be set at local level, by district managers, whose overlords could then truthfully deny the existence of a national eugenic policy' (4).

In this scenario not only patient autonomy but also clinical autonomy has given way. In order to restore it, arguments must be found against this threat. What I am suggesting is that it is incorrect to do this by arguing against eugenics as such. In fact Clarke himself says: 'There certainly is a role for public health genetics' (4). I would go further and suggest that there is a logical connection between a genetics counselling service and public health genetics (the latter being a term with fewer negative associations than eugenics).

But the fact that the genetic health of the population is an objective does not license any means whatever towards it, such as pressurising people to make particular choices.

\section{Reproductive autonomy}

A possible objection to the above would be to argue that autonomy, in particular reproductive autonomy, is itself the outcome sought. In other words, the aim of the service is the facilitation of reproductive choice. But then the question arises - why these choices? What about sex choice? Should terminations be offered, on autonomy grounds, to parents who want only male children? Clarke himself says 'no' to choices of blue-eyed girls (2).

There is an important difference between saying that an objective must be pursued by means compatible with autonomy, and saying that autonomy is itself the objective. The goals of the service set limits to the options available.

The autonomy model tells us who should be allowed to choose, not what the objectives should be. The objectives operate within the constraints set by autonomy. It is difficult to conceive of circumstances in which the potential benefit of pressurising a woman into a termination would outweigh the potential harm to her, and to the clinician/client relationship.

\section{Outcomes}

What, then, is the measure of a successful outcome? If what is important is autonomous decision-making then what would be required would be some measure of the extent to which individuals feel that they have been helped by the service. Clarke mentions the importance of client satisfaction (4). One way of measuring this would be a questionnaire to establish the extent to which clients are satisfied. But it has been argued that autonomous decision-making itself cannot be the only criterion of success. If implications for genetic health are important, how can that be measured? A questionnaire would need to elicit the kind of considerations that influenced decisionmaking, not with a view to measuring success in terms of decisions made, but in order to measure the extent of awareness, brought about by genetics counselling, of factors relevant to genetic disease.

The fact that it has been argued that genetics counsellors must have an eye to the genetic health of the population does, however, raise a question about the extent and nature of the information that can and should be provided. Although it might be argued that the counsellor can convey impartial factual information it is not clear that this is the case. The decisions that people will have to make involve questions about the worthwhileness of lives that future people will live, and whether or not it can be said to be better that someone should not be born. These are philosophical questions. How can individuals be provided with the necessary information to make such choices? Should they be acquainted with the latest moves in the philosophical debate about the value of life?

It might be argued that this would not strictly speaking be genetics counselling. It is consistent with what has been argued here, however, that if there is concern for genetic health at a national level, and attempts to set national targets, then this information itself should be provided. This might be seen as 'pressurising', but it is arguably preferable to keeping such a policy secret and dressing up a service as promoting individual choice when in fact there are constraints on the choices on offer.

\section{Conclusion}

I have argued as follows:

1) An outcome measure for a medical genetics unit that equates success with the number of termina- 
tions performed is incompatible with the autonomy model of health care. The latter, and not the former, should be retained.

2) A concern for eugenics, however, is not incompatible with the autonomy model. The one relates to objectives; the other to means. Autonomy should not itself be seen as the objective because in fact there are reasons for limiting choice in this area.

3) The autonomy model tells us who should be allowed to choose but does not tell us what the objectives should be.

4) Given the specified objectives it is arguable that the kind of information conveyed in counselling should include what they are, beyond the immediate context of the individual client. Success consists in individuals making choices in the light of relevant genetic information. A suitable outcome measure would be a questionnaire measuring satisfaction with the service and the reasons given for choice.
Ruth F Chadwick, BPhil, MA, DPhil (Oxon), LLB (London) is Lecturer in Philosophy and Director of the Centre for Applied Ethics, University of Wales College of Cardiff.

\section{References}

(1) Clarke A. Genetics, ethics and audit. Lancet 1990; 335: 1145-1147.

(2) See reference (1) 1146.

(3) Chadwick R, Russell J. Hospital discharge of frail elderly people: social and ethical considerations. Ageing and society 1989; 9: 277-295.

(4) See reference (1) 1147.

(5) Clarke A. Molecular genetics in medicine: ethical implications. Paper presented to the Galton Institute symposium held in London, 1989 Sept 22.

(6) Williams B. The idea of equality. In: Williams B Problems of the self. Cambridge: Cambridge University Press, 1973. Williams does not himself use the 'internal-external' language. 